\title{
Wie kann Forschendes Lernen evaluiert werden?
}

\section{Janina Thiem und Christopher Gess}

Forschendes Lernen wird bislang nur an wenigen Hochschulen gezielt evaluiert. Um die Evaluationspraxis voranzubringen, gibt der vorliegende Beitrag einen Überblick über Evaluationen zum Forschenden Lernen. Laufende und abgeschlossene Evaluationen werden nach verwendeten Forschungsmethoden sowie nach der Zielgruppe der Evaluation systematisch dargestellt. Darüber hinaus werden Entwicklungspotenziale für die Evaluation von Forschendem Lernen aufgezeigt.

\section{$1 \quad$ Einleitung}

Mit der Ausrichtung auf die Kompetenzentwicklung von Studierenden, die im Zuge des Bologna-Prozesses erfolgte, haben alternative Lehrformate wie das problembasierte Lernen, das Lernen in Projekten und das Forschende Lernen Konjunktur (Metz-Göckel, Kamphans \& Scholkmann, 2012, S. 216). Im Sinne des „Shifts from Teaching to Learning“ (Barr \& Tagg, 1995) liegt die Aktivität in diesen Lehr-Lern-Formaten aufseiten der Lernenden, die ihr Wissen selbst entwickeln und in ihre individuelle Wissensstruktur überführen (Pasternack, 2017, S. 40). Auf diese Weise eignen sich die Studierenden Kompetenzen an, die über den reinen Wissenserwerb hinausgehen. 
Ursprünglich geht es bei Forschendem Lernen um ein Lernen durch Forschung oder durch Beteiligung an Forschung (Bundesassistentenkonferenz, 1970). So liegen die Besonderheiten des Forschenden Lernens - im Vergleich zu anderen aktivierenden Lehr-Lern-Formaten - darin, dass die Studierenden selbst (oder mit Unterstützung der Lehrenden) eine sie interessierende Frage- oder Problemstellung entwickeln und diese auf die Gewinnung neuer Erkenntnis ausgerichtet ist. Dieser Prozess kann - wie im problembasierten Lernen - von einem konkreten Fall oder Problem ausgehen, führt aber weiter, da beim Forschenden Lernen nicht praktische Produkte, sondern theoretische Einsichten und empirische Erkenntnisse im Zentrum stehen (Huber, 2004). Darüber hinaus soll Wissenschaft durch das Forschende Lernen als sozialer und emotionaler Prozess erfahrbar werden:

\begin{abstract}
„Denn das Wichtige am Prinzip des Forschenden Lernens ist die kognitive, emotionale und soziale Erfahrung des ganzen Bogens, der sich vom Ausgangsinteresse, den Fragen und Strukturierungsaufgaben des Anfangs über die Höhen und Tiefen des Prozesses, Glücksgefühle und Ungewissheiten, bis zur selbst (mit-)gefundenen Erkenntnis oder Problemlösung spannt“" (Huber, 2004, S. 33).
\end{abstract}

Die wissenschaftstheoretische (und -politische) Begründung für das Forschende Lernen ist dabei keine ausschließlich idealistische (im Humboldt'schen Sinne), sondern vielmehr eine funktionalistische (Funktion der Hochschulbildung für das vornehmlich außerwissenschaftliche Beschäftigungssystem) (Pasternack, 2017). So zielt das Forschende Lernen nicht nur auf eine Förderung von Forschungskompetenzen und metakognitiven Kompetenzen sowie auf die Entwicklung einer forschenden Haltung (Huber, 2004; Gess, Deicke \& Wessels, 2017), sondern soll auch durch die Vermittlung von sogenannten Schlüsselkompetenzen wie Problemlösungs-, Analyse- und Kommunikationsfähigkeit zur allgemeinen Berufsqualifizierung der Studierenden beitragen (Huber, 2004; Wissenschaftsrat, 2001, 2006, 2015; Schubarth \& Speck, 2014). Angesichts der zunehmenden Ähnlichkeiten zwischen Forschungsprozess einerseits und problemlösendem beruflichen Handeln andererseits schlägt Pasternack eine Brücke zwischen beiden Begründungen:
„Um in den sich ergebenden beruflichen Situationen sicher handeln zu können, wird wissenschaftlich basierte Urteilsfähigkeit - das heißt die Befähigung, komplexe Sachverhalte methodisch geleitet und kritisch zu analysieren und bewerten - sowie eine explizit darauf gründende Handlungsfähigkeit benötigt. [...] , Das Lernziel lautet intelligentes Wissen aufzubauen, das die Übertragung von Lösungsstrategien auf neue Situationen ermöglicht' (Schumacher, 2009, S. 883)“(Pasternack, 2017, S. 41).

Die Erwartungen an das Forschende Lernen im Hinblick auf die Aneignung vielfältiger Kompetenzen sind somit sehr hoch. Damit verbunden ist auch ein 
hoher Anspruch an die Evaluation von Forschendem Lernen, denn je breiter und differenzierter die Wirkungserwartungen sind, desto mehr Voraussetzungen hat die Wirkungsanalyse hinsichtlich der Operationalisier- und Messbarkeit und der Interpretation von Ergebnissen zu erfüllen. Da so unterschiedliche und teils abstrakte Kompetenzziele verfolgt werden, ist die Evaluation mit einigen Herausforderungen verbunden. Trotz der wachsenden Anzahl von Projekten, die Forschendes Lernen an deutschen Hochschulen fördern - etwa im Rahmen des Qualitätspakts Lehre, der Qualitätsoffensive Lehrerbildung oder der Einführung von Forschungsanteilen im Praxissemester im Lehramt - wird Forschendes Lernen über gängige Lehrevaluationen hinaus bislang nur an wenigen Hochschulen gezielt evaluiert. Dies überrascht, da zum einen durch den Projektträger die evaluative Begleitung dieser Maßnahmen vorgeschrieben ist (z. B. BMBF, 2013) und zum anderen - auf einer allgemeineren Ebene - der Nachweis von Qualitätssicherungsmaßnahmen eine notwendige Voraussetzung zur erfolgreichen Akkreditierung von Studienprogrammen ist (Braun \& Hannover, 2012).

Ziel des vorliegenden Beitrags ist deshalb, einen Überblick über Evaluationsbemühungen zum Forschenden Lernen zu geben und auf bereits etablierte Evaluationskonzepte zu verweisen, an denen sich Praktikerinnen und Praktiker orientieren können. Dafür werden bestehende Evaluationen zum Forschenden Lernen in einem Systematisierungsraster verortet, das nach verwendeten Forschungsmethoden sowie nach der Zielgruppe der Evaluation differenziert. Im Ausblick werden abschließend Lücken identifiziert und Entwicklungspotenziale aufgezeigt.

\section{$2 \quad$ Was macht eine Evaluation aus?}

Das Ziel von Evaluationen ist die Sammlung von Informationen und deren Bewertung im Hinblick auf ein Evaluationsobjekt (z. B. eine Maßnahme, ein Projekt oder Programm), um Entscheidungen zu treffen (Stockmann, 2016, S. 36). Diese Definition reicht jedoch nicht aus, um Evaluation vom "Alltagshandeln“ zu unterscheiden, wonach irgendetwas von irgendjemandem nach irgendwelchen Kriterien in irgendeiner Weise bewertet wird (Kromrey, 2003, S. 107). Bei einem solchen Vorgehen kann derselbe Sachverhalt von verschiedenen Personen sehr unterschiedlich oder gar gegensätzlich bewertet werden. Professionelle, wissenschaftlich durchgeführte Evaluationen sind dadurch gekennzeichnet, dass 
- sie sich auf einen klar definierten Evaluationsgegenstand beziehen,

- empirische (sozialwissenschaftliche) Forschungsmethoden zur Informationsgewinnung nutzen,

- die Bewertung anhand explizit festgelegter, intersubjektiv nachprüfbarer Kriterien erfolgt,

- systematische (vergleichende Verfahren) zur Informationsbewertung verwendet werden,

- die Durchführung von in der Regel dafür besonders befähigten Personen (Evaluatoren) erfolgt,

- sie das Ziel verfolgen, den Nutzen (Wert) eines Evaluationsobjektes zu bestimmen, um Entscheidungen treffen zu können (Stockmann, 2016, S.36; Kromrey, 2005).

Mit anderen Worten bedeutet das, dass für jede professionell durchgeführte Evaluation zu klären ist, was wozu anhand welcher Kriterien von wem wie evaluiert wird.

Dabei ist Evaluation im professionellen Sinn angewandte Sozialwissenschaft (Kromrey, 2005). Das Besondere der Evaluation liegt darin, dass sie - im Unterschied zur wissenschaftlichen (Grundlagen-)Forschung - keinen Selbstzweck darstellt, indem sie nicht (ausschließlich) dem Erkenntnisinteresse verpflichtet ist, sondern einen Nutzen stiften soll (Stockmann, 2002). Während sich Grundlagenforschung bei allen zu treffenden Entscheidungen ausschließlich an wissenschaftlichen Kriterien zu orientieren hat (insbesondere Erkenntnisfortschritt als Selbstzweck, Gültigkeit, Zuverlässigkeit, Generalisierbarkeit der Ergebnisse), ist darüber hinaus für Evaluationen als Form der anwendungsorientierten Forschung die Berücksichtigung von außerwissenschaftlichen Erkenntnisinteressen und Verwertungskontexten zentral (Kromrey, 2003). Für die angewandte Sozialwissenschaft sind also beide Prinzipien relevant. Ein weiterer Unterschied zur Grundlagenforschung liegt darin, dass Grundlagenforschung „sich irren“ darf (Kromrey, 2003, S. 98). Hypothesen dürfen sich im Laufe der Forschung als falsch erweisen und können damit sogar den Grundstein für neue Erkenntnisse legen. Dies gilt nicht für die angewandte Sozialforschung, die Handlungswissen für die Praxis liefert. Da falsche Interpretationen, die zum Beispiel aufgrund fehlerhafter Forschungsdaten oder falscher Prämissen gezogen werden, reale Konsequenzen für die Betroffenen haben, ist eine sorgfältige Konzipierung des Forschungsdesigns essenziell. Das bedeutet, dass sich die Annahmen und Hypothesen bereits empirisch bewährt haben sollten und der Prozess der Erhebung, Auswertung und Interpretation aller Informationen methodisch abgesichert erfolgen sollte (Kromrey, 2003, S. 98).

In der Evaluationsforschung werden drei Funktionen von Evaluationen („Paradigmen“) analytisch unterschieden (Chelimsky, 1997; Kromrey, 2005): 
1. Erkenntnisgeleitete Evaluationen („Forschungsparadigma“): Diese Form der Evaluation weist eine große Nähe zur sozialwissenschaftlichen (Grundlagen-) Forschung auf und kann als Bindeglied zwischen Theorie und Praxis angesehen werden (Kromrey, 2005). Es geht hier insbesondere um Wirkungsforschung, wofür die Frage nach der Kausalität zentral ist - also die Prüfung, ob die beobachteten Wirkungen eine Folge der zu evaluierenden Maßnahmen sind oder ob sie auf andere Faktoren zurückzuführen sind. Ein Ziel von erkenntnisgeleiteten Evaluationen kann darin liegen, aus den gewonnenen Erkenntnissen Steuerungsentscheidungen abzuleiten (Stockmann, 2016).

2. Ausübung von Kontrolle („Kontrollparadigma“): Hier ist anhand von objektivierbaren Erfolgskriterien (z. B. Effektivität, Effizienz, Akzeptanz) zu prüfen, ob die festgelegten Ziele eines Programmes erreicht wurden; dies kann sich zum Besipiel auf die ordnungsgemäße Verausgabung von Finanzmitteln beziehen. Wie und nach welchen Kriterien die Erfolgskontrolle erfolgt, hängt vom Informationsbedarf der ausführenden und/oder der finanzierenden Instanz $a b$ (Kromrey, 2005).

Diese beiden Evaluationtypen sind durch mindestens drei interdependente Dimensionen gekennzeichnet: Ziele, Maßnahmen und Effekte. Diese Dimensionen sind zu präzisieren und zu operationalisieren. Dabei sind Ziele von Maßnahmen (als Instrumente zu deren Erreichung) abzugrenzen; die vorgesehene Umsetzung ist ebenso zu konkretisieren wie das, was das Vorhaben bewirken soll (und möglicherweise unbeabsichtigt bewirken kann) (Kromrey, 2005). Im Gegensatz zu einer Laborsituation können die Dimensionen nicht von Umwelteinflüssen abgeschirmt werden.

3. Auslösung von Entwicklungs- und Lernprozessen („Entwicklungsparadigma“): Die Problemstellung und das Erkenntnisinteresse der Evaluation sind hier - im Vergleich zum Forschungs- und zum Kontrollparadigma - anders ausgerichtet. So steht in dieser Form der Evaluation das Lernen im Vordergrund. Die Evaluationen werden deshalb in der Regel formativ, also programmgestaltend, durchgeführt. Die Lern- und Entwicklungsprozesse können sowohl auf der Basis von Best Practices als auch durch „fehlerhafte“ Maßnahmen erfolgen (Stockmann, 2016).

Dass die Paradigmen in der Praxis nicht immer trennscharf abgrenzbar sind, zeigt der aus der amerikanischen Bildungsforschung stammende Ansatz des Design-based Research (Brown, 1992; Collins, 1992), der neben der Evaluierung von Veränderungsmaßnahmen in seiner konzeptionellen Ausrichtung auch gezielt die (Weiter-)Entwicklung dieser Maßnahmen einplant und damit die Entwicklung und 
den Test einer Intervention berücksichtigt (Gess, Rueß \& Deicke, 2014). Zentral für den Ansatz ist, dass die Entwicklung von Interventionen zwar auf Theorien basiert und den aktuellen Forschungsstand berücksichtigt, jedoch immer bezogen auf die Lösung eines konkreten, praktischen Problems erfolgt (Reinmann, 2005).

Die Festlegung auf ein Evaluationsparadigma hat Auswirkungen auf das Design und die Durchführung der Evaluation. Abhängig von dem Verwendungszweck und dem damit verbundenen Erkenntnisinteresse können Evaluationen mehr formativ, das heißt aktiv gestaltend, prozessorientiert und kommunikationsfördernd angelegt sein, oder mehr summativ, das heißt zusammenfassend, bilanzierend und ergebnisorientiert (Stockmann, 2016).

Forschungsmethodisch kann Evaluation auf das gesamte Spektrum der empirischen Sozialforschung zurückgreifen. Zu unterscheiden ist hier zwischen der qualitativen und quantitativen Sozialforschung (z.B. Bryman, 2001; Döring \& Bortz, 2016; Creswell, 2014; Hitzler, 2016) sowie der Mixed-Methods-Forschung, die unterschiedliche Kombinationen qualitativer und quantitativer Forschung umfasst (Johnson, Onwuegbuzie \& Turner, 2007); gerade bei den qualitativen Forschungsmethoden existiert eine Vielzahl methodologischer und methodischer Ansätze (Mruck, Cisneros Puebla \& Faux, 2005). Während auf die Methodologien im vorliegenden Beitrag schon aus Platzgründen nicht vertieft eingegangen werden kann, ist für die angestrebte Systematisierung von Evaluation zumindest der Blick auf mögliche Erhebungsmethoden von Interesse. Mey (2016) unterscheidet in der qualitativen Sozialforschung zwischen Interview, Gruppendiskussion, teilnehmende Beobachtung, Videografie, Analyse von Dokumenten, Artefakten, Bildmaterial und Interaktionen.

\section{Evaluationen von Forschendem Lernen}

Im Hinblick auf Evaluationen zum Forschenden Lernen wurde die Leitfrage verfolgt, für welche Evaluationstypen bereits Evaluationskonzepte und -instrumente vorliegen. Zu diesem Zweck wurde eine offene Abfrage an deutschen Hochschulen (Universitäten und Fachhochschulen) durchgeführt, ob und wie dort Forschendes Lernen evaluiert wird. In dem Fragebogen wurde abgefragt, was aus dem Bereich des Forschenden Lernens vor Ort mit welchem Ziel wie evaluiert wird. Dabei waren sowohl spezifisch ausgerichtete Evaluationen, die sich ausschließlich auf das Forschende Lernen beziehen, von Interesse als auch allgemeine Studiengangs- und Lehrveranstaltungsevaluationen - sofern diese spezifische Fragestellungen zum Forschenden Lernen berücksichtigen. 
Für die Abfrage wurden die Verteiler der Arbeitsgemeinschaft Forschendes Lernen in der Deutschen Gesellschaft für Hochschuldidaktik (dghd) und des Qualitätspakt-Lehre-Begleitforschungsprogramms ForschenLernen genutzt. Die Abfrage wurde ab Sommer 2015 durchgeführt und laufend ergänzt. Insgesamt haben sich 14 Hochschulen gemeldet, hiervon führen sechs Hochschulen spezifische Evaluationen zum Forschenden Lernen durch (Humboldt-Universität (HU) zu Berlin, Universität Bremen, Universität Duisburg-Essen, Universität Hohenheim, Carl von Ossietzky Universität Oldenburg, Fachhochschule Potsdam). An der Freien Universität (FU) Berlin erfolgt eine Abfrage von forschungsbezogenen Kompetenzen sowie von Forschungsbezügen der Studiengänge im Rahmen einer Evaluation der konsekutiven, nicht lehramtsbezogenen Master-Studiengänge.

Die Evaluationen werden durchgeführt mit dem Ziel der Weiterentwicklung von spezifischen Formaten des Forschenden Lernens (HU Berlin, Duisburg-Essen, Hohenheim, Oldenburg, Potsdam) oder von gesamten Studiengängen (FU Berlin, Bremen) sowie mit dem Ziel der Profilbildung der gesamten Universität (Bremen, Oldenburg). In allen evaluierten Vorhaben wird die Definition Forschenden Lernens nach Huber (2009) zugrunde gelegt.

Um einen Überblick über die Evaluationslandschaft im Forschenden Lernen geben zu können, werden diese Evaluationen in einem Systematisierungsraster dargestellt (siehe Tab. 1). Dafür wurden sie den drei Evaluationsparadigmen zugeordnet. Da diese in der Empirie nicht immer trennscharf abzugrenzen sind, wurden einzelne Vorhaben zum Teil zwei Paradigmen zugeordnet. In einem weiteren Schritt wurden die in der Evaluation berücksichtigten inhaltlichen Dimensionen des Forschenden Lernens herausgearbeitet sowie die für die Evaluation verwendeten Methoden dargestellt. Das Systematisierungsraster berücksichtigt ebenfalls, an welche Zielgruppe sich die Evaluation richtet. Um die Einordnung der jeweiligen Evaluation in das Raster zu validieren, wurden kurze Interviews mit den Evaluationsverantwortlichen durchgeführt. In der Tabelle sind nur jene Evaluationen der Hochschulen berücksichtigt, die sich auf die genannte Anfrage gemeldet haben. Die Systematisierung erhebt keinen Anspruch auf Vollständigkeit. 


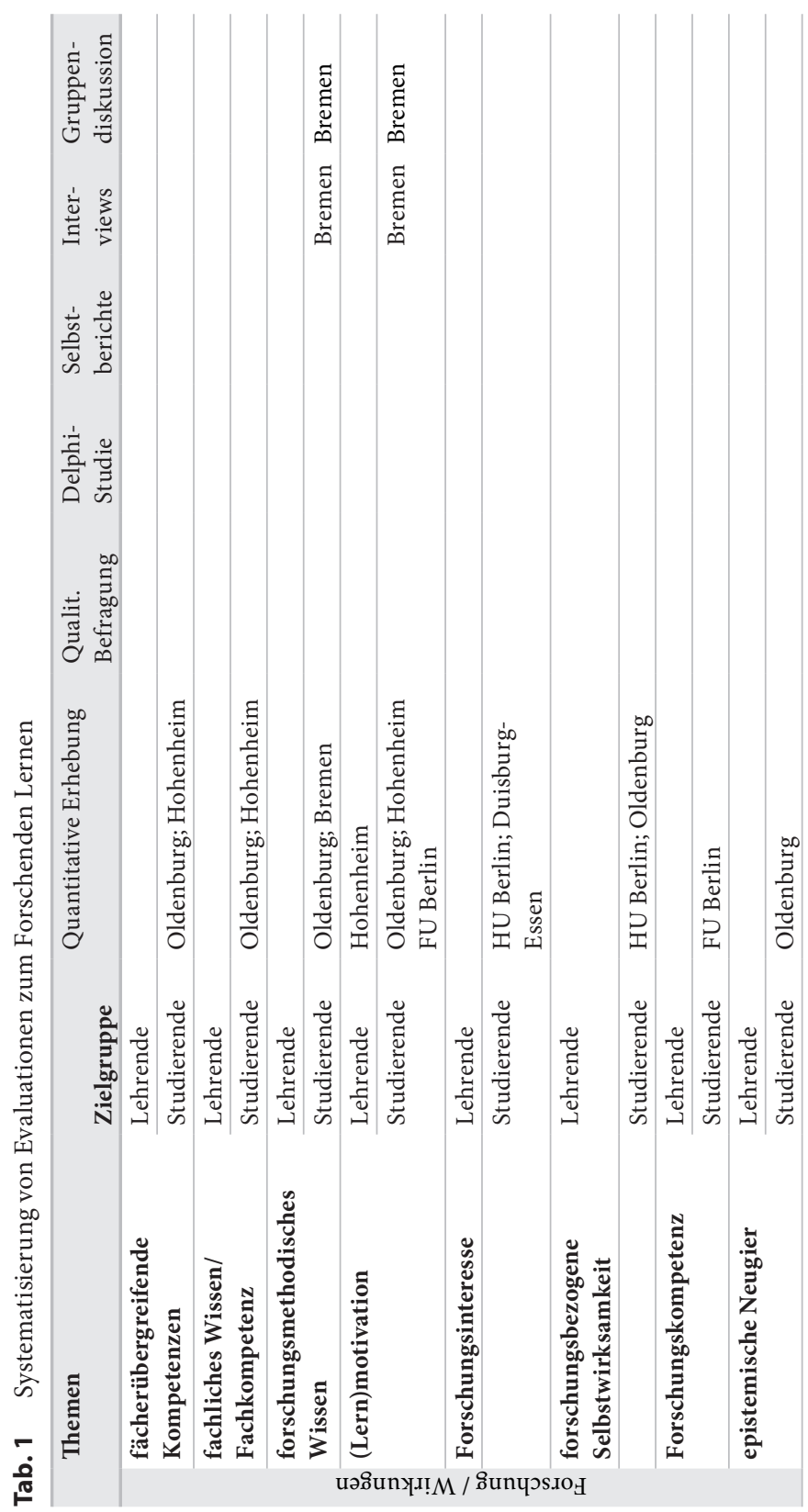




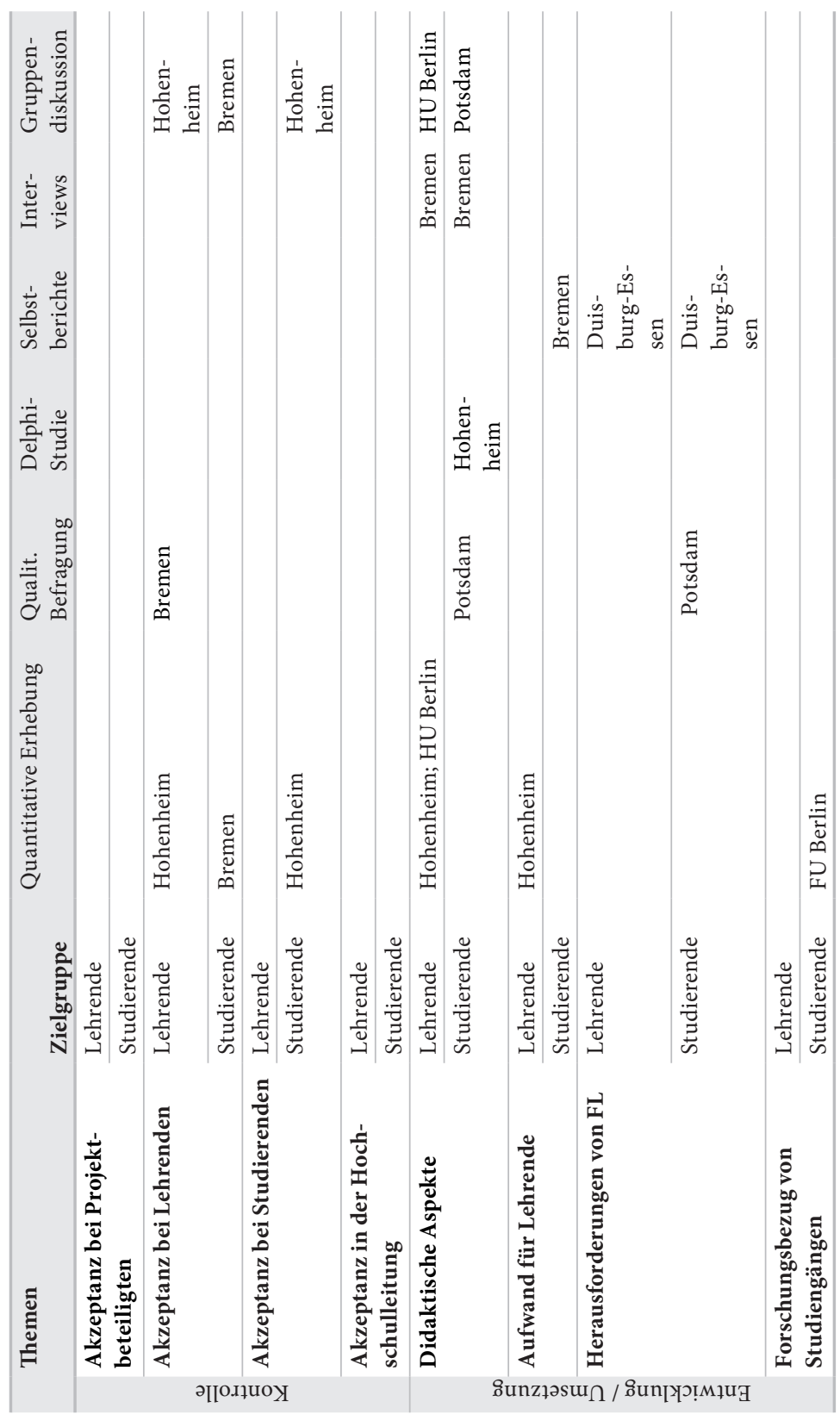




\subsection{Evaluationen von Forschendem Lernen im Sinne des Forschungsparadigmas}

In diesem Paradigma ist insbesondere die Analyse von Wirkungen von Interesse. So geht es in den vorliegenden Evaluationen dieses Paradigmas in erster Linie um Untersuchungen von Lernerfolgen oder Kompetenzzuwächsen durch Forschendes Lernen. Größtenteils erfolgt dies anhand quasi-experimenteller Forschungsdesigns mittels Prä-Post-Messungen in quantitativen Befragungen. Lediglich die Universität Bremen führt eine qualitative Untersuchung mittels Interviews und Gruppendiskussionen durch. Sie wird dem Forschungsparadigma zugeordnet, da Selbsteinschätzungen der Studierenden über ihr forschungsmethodisches Wissen und ihre Lernmotivation im Zeitverlauf eines an Forschendem Lernen ausgerichteten Studiengangs untersucht wurden (Kaufmann \& Koch, 2015; Kaufmann \& Schelhowe, 2017). An der Freien Universität Berlin erfolgt im Rahmen einer Studiengangsevaluation eine retrospektive Einschätzung zum Erwerb forschungsbezogener Kompetenzen.

In den Wirkungsanalysen werden die folgenden Zielkonstrukte betrachtet:

- forschungsmethodisches Wissen sowie allgemeine forschungsbezogene Kompetenzen (nur retrospektiv);

- Forschungsinteresse, forschungsbezogene Selbstwirksamkeit und weitere forschungsbezogene affektiv-motivationale Konstrukte;

- epistemische Neugier, (Lern-)Motivation und weitere affektiv-motivationale wie kognitive fächerübergreifende Kompetenzen;

- fachliches Wissen sowie allgemeine Fachkompetenz.

Während sich die beschriebenen Evaluationen an der Humboldt-Universität zu Berlin, der Freien Universität Berlin sowie an den Universitäten Oldenburg und Duisburg-Essen ausschließlich an Studierende richten, werden im Rahmen der Hohenheimer sowie der Bremer Evaluationen auch Lehrende einbezogen.

Für die Messung der Zielkonstrukte wurden in den Evaluationen zum Teil eigens psychometrische Skalen entwickelt und validiert (z. B. forschungsbezogene Selbstwirksamkeit, Forschungsinteresse an der HU Berlin, siehe Deicke, Gess \& Rueß, 2014; Gess, Rueß \& Deicke, 2014) oder etablierte Skalen übernommen und gegebenenfalls angepasst. Es fällt auf, dass ein für das Forschende Lernen zentrales Konstrukt, die Forschungskompetenzen, in den genannten Wirkungsanalysen - mit Ausnahme einer retrospektiven Erhebung von selbsteingeschätzten Forschungskompetenzen (FU Berlin) - nicht berücksichtigt wird. 


\subsection{Evaluationen von Forschendem Lernen im Sinne des Kontrollparadigmas}

Im Zentrum dieses Evaluationsparadigmas steht die Erfolgskontrolle. Zur Systematisierung ist hier zunächst zu klären, wie der Erfolg in dem jeweiligen Vorhaben definiert wird. Bei den Evaluationen von Forschendem Lernen, die diesem Paradigma zugeordnet werden können, handelt es sich ausschließlich um Akzeptanzstudien, die an den Universitäten Hohenheim und Bremen durchgeführt werden. Erfolg wird hier demnach mit Akzeptanz bei relevanten Zielgruppen gleichgesetzt.

Die betrachteten Evaluationen richten sich sowohl an Studierende (Hohenheim, Bremen) als auch an Lehrende (Hohenheim) und folgen qualitativen (Bremen, Hohenheim) und quantitativen Ansätzen (Hohenheim). Während die Akzeptanzuntersuchung in Bremen im Rahmen der Studiengangsevaluation erfolgt, handelt es sich bei der Hohenheimer Analyse um eine einmalig durchgeführte Studie zur Einschätzung der Akzeptanz des Projektes „Humboldt reloaded“ (Voeth, Kienzle \& Heuchemer, 2015). Im Rahmen der Hohenheimer Studie wurden nicht nur Studierende und Lehrende befragt, die an dem Projekt teilgenommen bzw. Projektveranstaltungen durchgeführt oder betreut haben, sondern auch „Non-User“. Dies hatte das Ziel, nach den Quellen möglicherweise mangelnder Akzeptanz zu suchen.

\subsection{Evaluationen Forschenden Lernens im Sinne des Entwicklungsparadigmas}

In diesem Paradigma ist es das Ziel, Lern- und Entwicklungsprozesse zu stimulieren. Entsprechende Evaluationen werden deshalb in der Regel formativ durchgeführt, also prozessbegleitend. Im Entwicklungsparadigma ist die Einbindung und ausführliche Rückmeldung der beteiligten Agierenden wichtig (Stockmann, 2016), weshalb insbesondere qualitative Methoden eingesetzt werden.

In den Evaluationen von Forschendem Lernen, die dem Entwicklungsparadigma zugeordnet werden können, steht überwiegend die didaktische Umsetzung von Lehrveranstaltungen im Format des Forschendem Lernen im Zentrum, beispielsweise die genutzten Lehrmethoden oder die Schwerpunkte innerhalb der Seminare auf bestimmte Phasen des Forschungsprozesses. Andere Evaluationen betrachten die Herausforderungen von Forschendem Lernen, beispielsweise den Aufwand für die Lehrenden, und suchen nach Möglichkeiten, Forschendes Lernen in der Breite attraktiver zu gestalten. Dies wurde an der Universität Hohenheim umgesetzt.

Auffallend ist zudem, dass einige Evaluationen des Forschungsparadigmas auch formative und qualitative Elemente im Sinne des Entwicklungsparadigmas aufweisen 
und insofern beiden Paradigmen zuzuordnen sind. So kombiniert beispielsweise die Humboldt-Universität zu Berlin Wirkungsanalysen mit Gruppendiskussionen unter Lehrenden, um Empfehlungen zur didaktischen Umsetzung von Forschendem Lernen geben zu können (Gess, Rueß \& Deicke, 2014).

\section{$4 \quad$ Zusammenfassung und Ausblick}

In deutschen Hochschulen wurden in den letzten Jahren einige Evaluationen zum Forschenden Lernen durchgeführt, wobei teilweise sehr unterschiedliche Evaluationsdesigns angelegt wurden. Am häufigsten wurden quantitative Wirkungsanalysen mittels Selbsteinschätzungen von Studierenden, qualitative Interviews und Gruppendiskussion zur didaktischen Umsetzung von Forschendem Lernen und Akzeptanzstudien konzipiert und realisiert. Sie sind jeweils unterschiedlichen Evaluationsparadigmen zuzuordnen und verfolgen unterschiedliche Ziele. Mit dem Beitrag werden somit erstmals systematisch Informationen von vorliegenden Evaluationskonzepten und -instrumenten zum Forschenden Lernen bereitgestellt, die beispielsweise von interessierten Hochschulen genutzt werden können, um eigene Evaluationen zu diesem Thema durchzuführen.

Eine Folge der Diversität von Evaluationsdesigns ist, dass sich die Evaluationsergebnisse nicht vergleichen oder zusammenführen lassen. Wird Forschendes Lernen an zwei Hochschulen wiederholt entlang zweier sehr spezifischer Designs evaluiert und modifiziert, entwickeln sich an diesen Hochschulen in der Folge sehr unterschiedliche Praktiken. Dies ist einerseits wünschenswert, da auf diese Weise umfassende Erfahrungen mit der Lehr-Lern-Form gesammelt werden. Andererseits könnte dies jedoch dazu führen, dass der interuniversitäre Austausch zur Lehr-Lern-Form versiegt. Innerhalb dieses Zielkonflikts wäre es daher sinnvoll, zumindest einen Austausch an Evaluationsdesigns und Instrumenten zu ermöglichen. Bei der Entwicklung eines Evaluationsdesigns rund um Forschendes Lernen sollte daher nach Möglichkeit auf bereits entwickelte Verfahren und Instrumente zurückgegriffen und der Austausch mit Hochschulen gesucht werden, die bereits entsprechende Evaluationen durchgeführt haben. Ein solches Vorgehen erleichtert wiederum sowohl den thematischen Austausch zwischen den Hochschulen als auch den Vergleich von Evaluationsergebnissen.

Davon unbenommen sollten weitere Evaluationsdesigns und Instrumente entwickelt werden. So werden bei Betrachtung bestehender Designs einige Lücken deutlich. Die größte Lücke besteht vermutlich bei der Entwicklung von Forschungskompetenzen, immerhin ein zentrales Ziel von Forschendem Lernen. Zudem wurde 
der Kompetenzerwerb beim Forschenden Lernen zum Zeitpunkt der Abfrage an keiner Hochschule gemessen. Ebenfalls unbeachtet bleiben die Wirkungen für die Lehrenden, beispielsweise hinsichtlich ihrer Einstellungen gegenüber Studierenden sowie ihrer lerntheoretischen Überzeugungen oder ihrer hochschuldidaktischen Bedarfe. Zumindest im Hinblick auf die ersten beiden Lücken wurden in den letzten Jahren zwei Instrumente entwickelt, mit denen Forschungskompetenzen erhoben, teilweise sogar gemessen werden können: ein Fragebogen zur Erfassung fachkulturübergreifender studentischer Forschungskompetenzen (Böttcher \& Thiel, 2016) sowie ein Test zur Messung sozialwissenschaftlicher Forschungskompetenz (Gess, 2018):

- Selbsteinschätzung fachkulturübergreifender Forschungskompetenzen: Der von Böttcher und Thiel entwickelte Fragebogen dient dazu, die Förderung von selbsteingeschätzten studentischen Forschungskompetenzen in forschungsbezogenen Lehr-Lern-Formaten zu evaluieren (Böttcher \&Thiel, 2016, 2018). Das Instrument basiert auf einem generischen Modell fachkulturübergreifender studentischer Forschungskompetenzen (Thiel \& Böttcher, 2014). Es besteht aus insgesamt fünf Dimensionen und umfasst vier Kompetenzbereiche, die sich an der Logik des Forschungsprozesses orientieren (Böttcher \& Thiel, 2016). Dabei handelt es sich um die fachunabhängigen Dimensionen Recherche-, Methoden-, Reflexions- und Kommunikationskompetenzen, die um die Dimension des fachlichen Wissens ergänzt werden. Für jede der Dimensionen wurden mehrere Facetten operationalisiert. In konfirmatorischen Faktorenanalysen konnte die angenommene Modellstruktur von fünf Dimensionen weitgehend bestätigt werden.

- Messung sozialwissenschaftlicher Forschungskompetenzen: Der von Gess entwickelte Kompetenztest erlaubt es, die Entwicklung von forschungsbezogenem Wissen im Studienverlauf zu erheben (Gess, 2018). Es kann in allen Lehrformaten in sozialwissenschaftlichen Studiengängen eingesetzt werden. Der Test basiert auf einem Modell von Forschungskompetenz, das Forschungsprozesswissen, Methodenwissen und methodologisches Wissen umfasst (Gess, Rueß \& Blömeke, im Druck). In Validierungsanalysen konnte nachgewiesen werden, dass die Testwerte ein erlernbares, in sozialwissenschaftlichen Studienfächern vermitteltes und forschungsbezogenes Konstrukt wiedergeben (Gess, Geiger \& Ziegler, 2018). Da die vorliegenden Testaufgaben eine eindimensionale Interpretation der Testwerte ermöglichen, ist der Test in Veranstaltungen zu qualitativen wie auch zu quantitativen Methoden einsetzbar. Im Gegensatz zum Verfahren von Böttcher und Thiel erhebt der Test objektive Forschungskompetenz anstelle der Selbsteinschätzung von Forschungskompetenzen. 
Beide Instrumente ermöglichen es, Forschendes Lernen unter neuen Perspektiven zu evaluieren. Weitere Instrumente zu affektiv-motivationalen Forschungskompetenzen werden aktuell entwickelt (Wessels et al., 2018). Wir empfehlen, zukünftig Evaluationen, die im Feld des Forschungsparadigmas durchgeführt werden sollen, verstärkt an diesen Instrumenten auszurichten und Ergebnisse auszutauschen sowie Evaluationsstudien hochschulübergreifend oder als Längsschnittstudie anzulegen - eine Panelbefragung von Studierenden zum Forschen im Studium wird beispielsweise seit dem Wintersemester 2017/18 an der Universität Oldenburg durchgeführt.

Für Evaluationen, die das Entwicklungsparadigma berücksichtigen, empfehlen wir, die Wirkungen von Forschendem Lernen auf die Lehrenden zukünftig stärker als bislang zu untersuchen - auch im Hinblick auf begleitende Maßnahmen wie zum Beispiel hochschuldidaktische Weiterbildungsmaßnahmen zum Forschenden Lernen, die an der Technischen Universität Hamburg durchgeführt werden (Bulmann et al., 2018).

Schließlich empfehlen wir im Feld Kontrollparadigma, auf bisherigen Akzeptanzstudien aufzubauen und diese hochschulübergreifend durchzuführen. Auf diese Weise könnten unterschiedliche Umsetzungsformate und didaktische Begleitungssettings hinsichtlich der Akzeptanz verglichen werden.

\section{Literatur}

Barr, R. B. \& Tagg, J. (1995). From Teaching to Learning: A New Paradigm for Undergraduate Education. Change, 27(6), S.12-25.

Böttcher, F. \& Thiel, F. (2016). Der Fragebogen zur Erfassung studentischer Forschungskompetenzen: Ein Instrument auf der Grundlage des RMRK-W-Modells zur Evaluation von Formaten forschungsorientierter Lehre. In B. Berendt, A. Fleischmann, N. Schaper, B. Szczyrba \& J. Wildt (Hrsg.), Neues Handbuch Hochschullehre (I 2.11, S. 57-74). Berlin: DUZ Medienhaus.

Böttcher, F. \& Thiel, F. (2018). Evaluating research-oriented teaching: A new instrument to assess university students' research competences. Higher Education, 75(1), S. 91-110.

Braun, E. \& Hannover, B. (2012). Editorial. Zeitschrift für Erziehungswissenschaft, 15, S. 209-212.

Brown, A. L. (1992). Design Experiments: Theoretical and Methodological Challenges in Creating Complex Interventions in Classroom Settings. Journal of the Learning Sciences, 2(2), S. 141-178.

Bryman, A. (2001). Social reseach methods. Oxford: Oxford University Press.

Bulmann, U., Braun, S., Vosgerau, K., Ellinger, E. \& Knutzen, S. (2018). Combining research and teaching in engineering: Creating a didactic qualification programme on research-based 
learning for early stage researchers (Full Paper, Sefi Annual Conference). Copenhagen (accepted).

Bundesassistentenkonferenz (BAK) (1970). Forschendes Lernen - Wissenschaftliches Prüfen (Schriften der BAK, 5. Neudruck). Bielefeld: UVW.

Bundesministerium für Bildung und Forschung (BMBF) (2013). Richtlinien zur Förderung von Vorhaben zur Begleitforschung zum Qualitätspakt Lehre. BAnz AT 07.10.2013 B2.

Chelimsky, E. (1997). Thoughts for a new evaluation society: Keynote speech at the UK Evaluation Society conference in London 1996. Evaluation, 3(1), S.97-109.

Collins, A. (1992). Towards a design science of education. In E. Scanlon \& T. O'Shea, T. (Hrsg.), New directions in educational technology (S. 15-22). Berlin: Springer.

Creswell, J. W. (2014). Research design: qualitative, quantitative and mixed methods approaches. Los Angeles: Sage.

Deicke, W., Gess, C. \& Rueß, J. (2014). Increasing Student's Research Interest through Research-Based Learning at Humboldt-University. Council on Undergraduate Research Quarterly, 35(1), S.27-33.

Döring, N. \& Bortz, J. (2016). Forschungsmethoden und Evaluation in den Sozial- und Humanwissenschaften (5. Aufl.). Heidelberg: Springer.

Gess, C. (2018). Entwicklung und Validierung eines Tests zur Messung sozialwissenschaftlicher Forschungskompetenz (Unveröffentlichte Dissertationsschrift).

Gess, C., Deicke, W. \& Wessels, I. (2017). Kompetenzentwicklung durch Forschendes Lernen. In H. A. Mieg \& J. Lehmann (Hrsg.), Forschendes Lernen: Wie die Lehre in Universität und Fach hochschule erneuert werden kann (S.79-90). Frankfurt: Campus.

Gess, C., Geiger, C. \& Ziegler, M. (2018). Social-scientific research competency: Validation of test score interpretations for evaluative purposes in higher education. European Journal of Psychological Assessment. S. 1-14.

Gess, C., Rueß, J. \& Blömeke, S. (2019). Fach- und paradigmenübergreifendes Modell der Forschungskompetenz in den Sozialwissenschaften. Zeitschrift für empirische Hochschulforschung. S. 7-27.

Gess, C., Rueß, J. \& Deicke, W. (2014). Design-based Research als Ansatz zur Verbesserung der Lehre an Hochschulen - Einführung und Praxisbeispiel. Qualität in der Wissenschaft, 8(1), S. 10-16.

Hitzler, R. (2016). Zentrale Merkmale und periphere Irritationen interpretativer Sozialforschung. Zeitschrift für Qualitative Forschung, 17(1_2), S. 171-184.

Huber, L. (2004). Forschendes Lernen: 10 Thesen zum Verhältnis von Forschung und Lehre aus der Perspektive des Studiums. die hochschule, 13(2), S. 29-49.

Huber, L. (2009). Warum Forschendes Lernen nötig und möglich ist. In L. Huber, J. Hellmer \& F. Schneider (Hrsg.), Forschendes Lernen im Studium (S.9-35). Bielefeld: UVW.

Johnson, R. B., Onwuegbuzie, A. J. \& Turner, L. A. (2007). Toward a definition of mixed methods research. Journal of Mixed Methods Research, 1(2), S. 112-133.

Kaufmann, M. E. \& Koch, H. (2015). Evaluierende Begleitforschung zum Umgang mit Heterogenität beim Forschenden Lernen in der Studieneingangsphase. In S. Harris-Hümmert, L. Mitterauer \& P. Pohlenz (Hrsg.), Heterogenität der Studierendenschaften: Herausforderung für die Qualitätsentwicklung in Lehre und Studium, neuer Fokus für die Evaluation? (S. 113-128) Bielefeld: UVW.

Kaufmann, M. E. \& Schelhowe, H. (2017). Forschendes Lernen als Lehrprofil von Hochschulen - am Beispiel der Universität Bremen. In H. A. Mieg \& J. Lehmann (Hrsg.), 
Forschendes Lernen: Wie die Lehre in Universität und Fachhochschule erneuert werden kann (S.392-400). Frankfurt: Campus.

Kromrey, H. (2003). Evaluation in Wissenschaft und Gesellschaft. Zeitschrift für Evaluation, 2(1), S. 93-116.

Kromrey, H. (2005). Evaluation - ein Überblick. In H. Schöch (Hrsg.), Was ist Qualität? Die Entzauberung eines Mythos (Bd. 6, S.31-85). Berlin: Wissenschaftlicher Verlag (Schriftenreihe Wandel und Kontinuität in Organisationen).

Metz-Göckel, S., Kamphans, M. \& Scholkmann, A. (2012). Hochschuldidaktische Forschung zur Lehrqualität und Lernwirksamkeit: Ein Rückblick, Überblick und Ausblick. Zeitschrift für Erziehungswissenschaft, 15, S.213-232.

Mey, G. (2016). Qualitative Forschung: Zu einem Über(be)griff und seinen (Ver)Wendungen. ZQF - Zeitschrift für Qualitative Forschung, 17(1-2), S. 185-197.

Mruck, K., Cisneros Puebla, C. A. \& Faux, R. (2005). Editorial: Über Zentren und Peripherien qualitativer Forschung. Forum Qualitative Sozialforschung, 6(3).

Pasternack, P. (2017). Was die Hochschulforschung zum Forschenden Lernen weiß. In H. A. Mieg \& J. Lehmann (Hrsg.), Forschendes Lernen: Wie die Lehre in Universität und Fachhochschule erneuert werden kann (S.37-44). Frankfurt: Campus.

Reinmann, G., (2005). Innovation ohne Forschung? Ein Plädoyer für den Design-Based Research-Ansatz in der Lehr-Lernforschung. Unterrichtswissenschaft, 33(1), S. 52-69.

Schubarth, W. \& Speck, K. (2014). Employability und Praxisbezüge im wissenschaftlichen Studium. Fachgutachten für die Hochschulrektorenkonferenz. Verfügbar unter https:// www.hrk-nexus.de/fileadmin/redaktion/hrk-nexus/07-Downloads/07-02-Publikationen/ Fachgutachten_Employability-Praxisbezuege.pdf [20.02.2019].

Schumacher, R. (2009). Was heißt es, etwas verstanden zu haben? Menschliches Lernen aus Sicht der Psychologie. Forschung \& Lehre, 16(12), S. 882-883.

Stockmann, R. (2002). Qualitätsmanagement und Evaluation - Konkurrierende oder sich ergänzende Konzepte? Zeitschrift für Evaluation, 2, S. 209-243.

Stockmann, R. (2016). Entstehung und Grundlagen der Evaluation. In D. Großmann \& T. Wolbring, (Hrsg.), Evaluation von Studium und Lehre: Grundlagen, methodische Herausforderungen und Lösungsansätze (S.27-56). Wiesbaden: Springer VS.

Thiel, F. \& Böttcher, F. (2014). Modellierung fächerübergreifender Forschungskompetenzen: Das RMKR-W-Modell als Grundlage der Planung und Evaluation von Formaten forschungsorientierter Lehre. In B. Berendt, A. Fleischmann, N. Schaper, B. Szczirba \& J. Wildt (Hrsg.), Neues Handbuch Hochschullehre (Griffmarke I 2.10, S. 109-124). Berlin.

Voeth, M., Kienzle, N. \& Heuchemer, A. (2015). Beurteilung der Akzeptanz von Humboldt reloaded an der Universität Hohenheim. Universität Hohenheim. Verfügbar unter https:// studium-3-0.uni-hohenheim.de/akzeptanzstudie [20.02.2019].

Wessels, I., Rueß, J., Jenßen, L., Gess, C. \& Deicke, W. (30.07.2018). Beyond Cognition: Experts' Views on Affective-Motivational Research Dispositions in the Social Sciences. Frontiers in Psychology, 9:1300. Verfügbar unter https://www.frontiersin.org/articles/10.3389/ fpsyg.2018.01300/full [20.02.2019].

Wissenschaftsrat (2001). Empfehlungen zur künftigen Struktur der Lehrerbildung (Drs. 5065/01). Verfügbar unter https://www.wissenschaftsrat.de/download/archiv/5065-01. pdf [20.02.2019].

Wissenschaftsrat (2006). Empfehlungen zur künftigen Rolle der Universitäten im Wissenschaftssystem (Drs. 7067-06). Verfügbar unter https://www.wissenschaftsrat.de/download/ archiv/7067-06.pdf [20.02.2019]. 
Wissenschaftsrat (2015). Empfehlungen zum Verhältnis von Hochschulbildung und Arbeitsmarkt (Drs. 4925-15). Verfügbar unter https://www.wissenschaftsrat.de/download/ archiv/4925-15.pdf [20.02.2019].

\section{Zur Autorin und zum Autor}

Dr. Janina Thiem ist Mitarbeiterin in der Internen Evaluation im Referat Studium und Lehre der Carl von Ossietzky Universität Oldenburg und für die Projektevaluation des Qualitätspakt-Lehre-Projekts „Forschungsbasiertes Lernen im Fokus (FLiF)“ zuständig. E-Mail: janina.thiem@uol.de

Dr. Christopher Gess hat ein Lehramtsstudium an der Universität Bielefeld mit dem ersten Staatsexamen sowie ein Masterstudium der Public Administration an der Columbia University (USA) abgeschlossen. Er arbeitete von 2012 bis 2017 im bologna. lab der Humboldt-Universität zu Berlin. Dort lag sein Forschungsschwerpunkt auf den Wirkungen Forschenden Lernens, insbesondere auf dem Forschungsinteresse und der Forschungskompetenz. Letzteres ist das Thema seiner 2019 abgeschlossenen Dissertation. E-Mail: christopher.gess@hu-berlin.de

Open Access Dieses Kapitel wird unter der Creative Commons Namensnennung 4.0 International Lizenz (http://creativecommons.org/licenses/by/4.0/deed.de) veröffentlicht, welche die Nutzung, Vervielfältigung, Bearbeitung, Verbreitung und Wiedergabe in jeglichem Medium und Format erlaubt, sofern Sie den/die ursprünglichen Autor(en) und die Quelle ordnungsgemäß nennen, einen Link zur Creative Commons Lizenz beifügen und angeben, ob Änderungen vorgenommen wurden.

Die in diesem Kapitel enthaltenen Bilder und sonstiges Drittmaterial unterliegen ebenfalls der genannten Creative Commons Lizenz, sofern sich aus der Abbildungslegende nichts anderes ergibt. Sofern das betreffende Material nicht unter der genannten Creative Commons Lizenz steht und die betreffende Handlung nicht nach gesetzlichen Vorschriften erlaubt ist, ist für die oben aufgeführten Weiterverwendungen des Materials die Einwilligung des jeweiligen Rechteinhabers einzuholen.

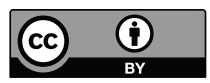

